\title{
ANÁLISES DE MÉTODOS DE AVALIAÇÃO DE PROJETOS
}

\author{
Analisys of design evaluation methods
Doris Catharine Cornelie Knatz KOWALTOWSKI' email I CV Lattes Paula Roberta Pizarro PEREIRA ii email CV Lattes

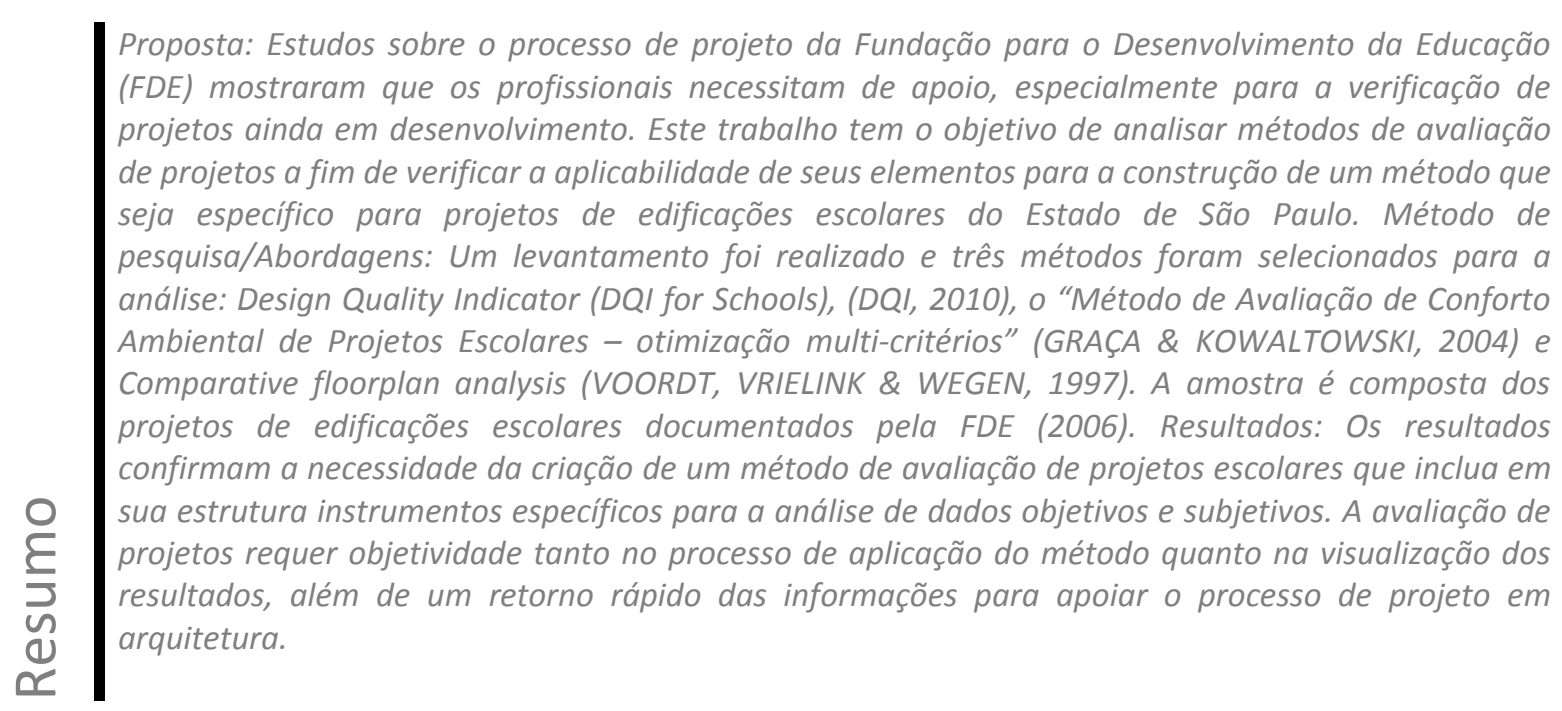

Palavras-chave: Arquitetura Escolar, Processo de Projeto, Avaliação de Projetos.

\begin{abstract}
Proposal: Studies on the State Foundation for the Development of Education (Fundação para o Desenvolvimento da Educação - FDE) design process show that professionals working for this foundation need support during the design process, especially to verify designs under development. This paper analyses design evaluation methods to verify the applicability for the construction of a specific method to evaluate school building designs in the State of São Paulo. Methods: A survey was held and three methods were selected for analysis: DQI for Schools (Design Quality Indicator), (DQI, 2010), the method called "Evaluation method for school building design with optimization of aspects of environmental comfort" (GRAÇA \& KOWALTOWSKI, 2004) and Comparative floorplan analysis (VOORDT, VRIELINK \& WEGEN, 1997). The sample is composed of school building designs documented by FDE (2006). Results: The results of the analysis of the three methods highlight the necessities for a school building design evaluation method that includes specific instruments to analyse both objective and subjective data within its structure. Design evaluation requires objectivity both in the application of the method and in result visualization, alongside a quick return of information to support the architectural design process. Essential also is the inclusion of specific design requirements for local conditions.
\end{abstract}

Palavras-chave: School Building Design, Design Process, Design Evaluation. 


\section{INTRODUÇÃO}

O ensino público brasileiro tem sido objeto de muitas discussões em razão dos índices insatisfatórios de desempenho obtidos pelos alunos (WERTHEIN, 2010). Dada a importância da educação para a sociedade, observa-se a necessidade de uma atuação multidisciplinar que vislumbre, ainda que em médio prazo, a melhoria da qualidade de ensino de forma geral, mas que deve incluir uma preocupação com o espaço físico, já que um número crescente de estudos demonstra a direta relação existente entre a qualidade da arquitetura escolar e o desempenho acadêmico dos alunos (KOWALTOWSKI, 2011; TARALLI, 2004). Assim, ao buscar dar voz à arquitetura como elemento significativo da determinação da qualidade do processo educacional, necessita-se focalizar a adequação do processo de trabalho dos projetistas às novas demandas exigidas. No Brasil as condições nem sempre ideais dos ambientes escolares apontam para o desafio aos arquitetos de se moverem das experiências de sucesso e de falha do passado para a criação de edifícios que sirvam às novas realidades e necessidades atuais e que sejam, ainda, flexíveis e adaptáveis às mudanças de um futuro próximo.

As discussões sobre qualidade do ambiente construído mostram que ela é resultado de um processo de projeto, de obra e de manutenção adequados, assim de um uso condizente com as suas funções. A qualidade do projeto em arquitetura depende da qualidade e experiência da equipe e da aplicação procedimentos metodológicos ao desenvolvimento de projeto, bem como das informações disponíveis para o desenvolvimento do projeto. Muitas pesquisas concentram-se na investigação das estratégias cognitivas de projeto e suas conseqüências na qualidade de determinado produto, firmando a etapa de elaboração do programa de necessidades e desenvolvimento do escopo como as mais importantes durante o processo de projeto (CHERRY, 1999; KRUGER \& CROSS, 2006). É importante também investigar a origem das falhas e estabelecer procedimentos que incluam em seu processo a tomada de decisões otimizada. Além disso, as etapas de avaliação de projeto não podem faltar permitindo uma condução mais segura desse processo. Pode-se considerar o processo de projeto como um número de atividades intelectuais básicas organizadas em fases de características e resultados distintos. Estas atividades são: análise, síntese, previsão, avaliação e decisão.
Para melhorar o processo de projeto e vencer obstáculos de sua complexidade são recomendados atualmente vários procedimentos. Na literatura internacional sobre arquitetura escolar recomenda-se valorizar a fase do programa de necessidades com participação de futuros usuários e discussão dos problemas a serem resolvidos e as possíveis soluções (SANOFF, 2001; NAIR \& FIELDING, 2005; DUDEK, 2007; FORD \& HUTTON, 2007 E LIPPMAN, 2010). Há necessidade de introduzir várias fases de avaliação permitindo a reflexão sobre decisões tomadas e a introdução de correções ao longo do processo de projeto. Finalmente, a Avaliação Pós-Ocupação (APO) e os registros dos erros e acertos de protótipos devem ser valorizados (PREISER et al.,1988).

No Brasil, as discussões sobre o ambiente escolar são abundantes e, em sua maioria, são pautadas em resultados de avaliações pósocupação - APO (KOWALTOWSKI et al., 2001, PIZARRO, 2005; ORNSTEIN \& MOREIRA, 2008; RHEINGANTZ et al., 2008; BLOWER \& AZEVEDO, 2010; KOWALTOWSKI, 2011, KOWALTOWSKI et al., 2011). No entanto, poucos trabalhos tratam da metodologia do processo de projeto (MÜLLER, 2007; FIGUEIREDO, 2009; DELIBERADOR, 2010; MOREIRA \& KOWALTOWSKI, 2009) e de avaliações de projetos (GRAÇA \& KOWALTOWSKI, 2004). Pesquisas em APO concentram-se nas falhas do ambiente físico pelas suas próprias evidências. A facilidade de aplicação de APOs permitiu o desenvolvimento de métodos e conceitos próprios, embora apresente realimentação de projetos lenta, sendo, desta forma, mais popular na academia que em escritórios de arquitetura. A avaliação de projetos arquitetônicos é feita de maneira informal pelos profissionais de projeto, sendo objeto de discussão mais freqüente para a crítica arquitetônica (KOWALTOWSKI et al., 2006).

Os resultados das APOs nas escolas do Brasil indicam que o conforto ambiental em edificações escolares, entre outros aspectos como a necessidade de adaptação do espaço para receber determinado projeto pedagógico e o desenvolvimento de projetos participativos, podem ser melhorados. Os estudos sobre o processo de projeto destes edifícios auxiliam no aumento da qualidade deste aspecto. No Estado de São Paulo a Fundação para o Desenvolvimento da Educação (FDE) é responsável por distribuir, coordenar e avaliar os projetos de edificações escolares 
desenvolvidos por escritórios terceirizados contratados. Disponibilizam-se aos escritórios o programa arquitetônico, previamente definido pela Secretaria da Educação, o levantamento topográfico e catálogos técnicos (componentes construtivos e modulação exigida), além de lista das normas que deverão ser consultadas. Recentemente a FDE também introduziu indicadores de sustentabilidade nos projetos através da certificação AQUA (FCAV, 2007).
Observa-se que os esquemas de processo de projeto nas escolas consideradas de alta qualidade são integrados e contém várias fases de avaliação de projeto antes da realização da obra. Desta forma, o processo de projeto eficaz exige a utilização de técnicas de avaliação de projetos que permitam garantir a obtenção dos resultados pretendidos e corrigir rapidamente desvios que venham a ser detectados antes da realização da obra.

\section{TÉCNICAS DE AVALIAÇÃO DE PROJETOS}

As técnicas de avaliação de projetos de arquitetura podem ser denominadas "métodos de avaliação" e "ferramentas de avaliação", sendo que a atribuição de significado ao uso destes termos geralmente não é padronizada na literatura por não possuírem uma terminologia descritiva precisa. Em relação à distinção entre o que método e do que é ferramenta, Campos (1992) afirma que o "método" é a seqüência lógica para alcançar a meta desejada e a ferramenta é o recuso utilizado no método.

Cole (2005) atribui significado aos termos "método" e "ferramenta", tendo em vista o conjunto de técnicas de avaliação de desempenho ambiental de edifícios. Para este, o termo "ferramenta de avaliação" é frequentemente usado para descrever as técnicas de avaliação que assistem a uma intenção específica. 0 autor inclui que as "ferramentas" são usadas para descrever técnicas que prevêem, calculam ou estimam a o desempenho de características ambientais de um edifício. Ferramentas podem ser compradas por profissionais de projeto ou outros e utilizadas quando e como forem julgadas necessárias.

Os "métodos de avaliação" possuem a atividade de avaliação como uma de suas funções principais, mas podem ser acompanhados por uma atividade de verificação antes de emitir a classificação do desempenho, incluir referências para utilização de outras ferramentas e podem oferecer programas de suporte educacional para profissionais de projeto. "Método de avaliação" geralmente apresenta "grades conceituais" reconhecíveis, que organizam ou classificam critérios de desempenho de uma maneira estruturada com pontos atribuídos ou pesos. Segundo o autor (COLE, 2005), dentro do contexto das técnicas de avaliação ambiental de edifícios, os "métodos de avaliação" são gerenciados e operados dentro de contextos organizacionais reconhecidos (ex: BREEAM e
LEED). Embora as partes de um método de avaliação possam ser usadas seletivamente por profissionais de projeto, a maior parte dos métodos envolve alguma forma de registro ou certificação. No caso das técnicas de avaliação de desempenho ambiental, esta característica representa uma distinção crítica entre "ferramentas de avaliação" e "métodos de avaliação". Além disso, ferramentas e métodos de avaliação têm diferentes curvas de aprendizado pelos seus usuários - a primeira se mostra mais íngreme que a segunda porque pode ser usada mais independentemente.

Tendo em vista as descrições das terminologias encontradas na literatura, esta pesquisa reúne em sua amostra três métodos de avaliação de projetos: DQI for Schools (DQI, 2010), e Comparative floorplan analysis (VOORDT, VRIELINK \& WEGEN, 1997) e o "Método de avaliação de conforto ambiental de projetos escolares - otimização multi-critérios" (GRAÇA \& KOWALTOWSKI, 2004). Estes são analisados a fim de conhecer os elementos que possam constituir um método de avaliação de projetos específico que auxilie o processo de projeto das escolas públicas do Estado de São Paulo. Os métodos são aplicados em projetos de edifícios escolares documentados (FDE, 2006). Na análise são descritos os seus conceitos, morfologia e forma de pontuação. São verificadas as facilidades e dificuldades da aplicação, características positivas e negativas em cada uma, a clareza dos resultados e o modo como cada uma realiza o retorno dos dados ao processo de projeto.

\subsection{Método de avaliação de conforto ambiental de projetos escolares - otimização multi-critérios}

O propósito principal da teoria de otimização é ajudar o projetista na seleção de um projeto que pertence a um conjunto de soluções viáveis ao 
problema, proporcionando direcionamento ao processo de decisão através da comparação entre os projetos e da seleção do "melhor". Esta situação de compromisso também é chamada de decisão ótima de "Edgeworth-Pareto", ou seja, na otimização multi-critério é necessário encontrar o conjunto de soluções de projeto que possuam compromisso e em conjuntos de soluções inferiores. $\mathrm{Na}$ prática a garantia de encontrar o melhor projeto é ilusória, na verdade se encontra o melhor projeto dentro de um conjunto existente de projetos (STADLER \& DAUER, 1992).

0 método de avaliação de conforto ambiental de projetos escolares - otimização multi-critério é utilizado para avaliar as condições de conforto acústico, térmico, luminoso e funcional em projetos de edificações escolares (GRAÇA \& KOWALTOWSKI, 2004). Pode ser dividido em três fases. Na primeira há a análise das dimensões dos terrenos em que estas escolas estão inseridas, de modo a permitir certa flexibilidade de decisões em projeto. A maioria dos projetos implantados nesses terrenos sofrem grandes restrições devido ao seu tamanho, prejudicando a tomada de decisão otimizada no anteprojeto. Sendo assim, considera-se que terrenos com dimensões menores que as estipuladas na Tabela 1 não fazem parte do conjunto de soluções viáveis para aplicação do método de avaliação.

Tabela 1. Dimensões mínimas de terreno e relação entre número de salas (em metros). Fonte: Graça \& Kowaltowski (2004).

\begin{tabular}{llll}
\hline Escolas & Largura (mínima) & Comprimento (mínimo) & Razão (L/C) (Mínima) \\
\hline $4-12$ salas & 36 & 43 & 0,36 \\
$13-15$ salas & 38 & 65 & 0,48 \\
$16-18$ salas & 40 & 70 & 0,48 \\
$19-21$ salas & 53 & 108 & 0,49 \\
\hline
\end{tabular}

A segunda fase consta de uma análise gráfica de variações tipológicas para os aspectos de conforto térmico, visual e acústico, onde os valores destas tipologias são expressos em graus de pertinência para cada variável encontrada. Os graus de pertinência foram definidos através de questionários respondidos por especialistas de cada área. Em uma escala de 5 pontos, os especialistas classificaram cada tipologia encontrada. Para o conforto térmico há sete configurações de salas de aula qualificadas considerando-se as posições das aberturas. Estas configurações podem se apresentar em oito tipos de orientação solar e ventilação predominante, representando um total de 56 variáveis de projeto. Para o conforto luminoso são consideradas as relações entre aberturas e orientação solar, totalizando 144 variáveis. Para o conforto acústico são consideradas a relação entre as salas de aula e as áreas de corredores e recreação, totalizando 8 variáveis (Figura 1). Para o conforto funcional analisa-se a distância entre as salas de aula e os banheiros, sendo que as medidas são também convertidas em graus de pertinência. A transformação das medidas em graus de pertinência considera a relação do tempo gasto para a locomoção de um ambiente para outro e o tempo da atividade realizada no ambiente. 0 conforto funcional não foi avaliado nesta pesquisa.

Por fim, há a escolha de uma solução de compromisso de projeto, já que os parâmetros de conforto interferem ente si. 0 critério para identificar as soluções inferiores é: soluções que possuem todas as avaliações de parâmetros de conforto com índices inferiores a outro projeto. Define-se por eliminação o critério de maximização de projeto que identifica as soluções de compromisso da seguinte maneira: soluções que possuem pelo menos uma avaliação de parâmetro de conforto com índice superior a outro projeto.

Inicialmente 34 escolas da amostra foram eliminadas nesta avaliação, algumas em função das dimensões de largura, outras, do comprimento, outras apresentaram razões abaixo do recomendado. 0 número de escolas da amostra é grande, portanto foi feita a transferência das plantas para um programa CAD (Computer Aided Design) e a criação de blocos em CAD representando cada tipologia e sua orientação correspondente, a fim de que tanto a análise de projeto quanto a verificação 
do grau de pertinência fosse facilitada e possível de ser executada diretamente na tela do CAD. Um exemplo de solução de compromisso é a escola \#65 da amostra. Seus valores de grau de pertinência são: 0,65 para conforto térmico, 0,65 para conforto luminoso e 0,71 para conforto térmico.

Um exemplo de melhoramento de uma soluçãoinferior pode ser aplicado na escola \#59 da amostra, que apresentou valores de grau de pertinência de 0,08 para conforto térmico, 0,04 para conforto luminoso e 0,50 para conforto acústico. Os baixos índices para conforto térmico e luminoso são decorrentes da orientação LesteOeste das salas de aula analisadas (Figura 2). Uma forma de aumentar os valores dos índices e, por conseqüência, a qualidade do projeto, seria utilizar o trecho marcado como "E" no projeto para dispor de salas com orientação Norte-Sul. Ressalta-se que os valores de conforto acústico também seriam alterados sofrendo uma pequena diminuição, passando a pertencerem à tipologia 6 do método (Figura 1 e Figura 2).

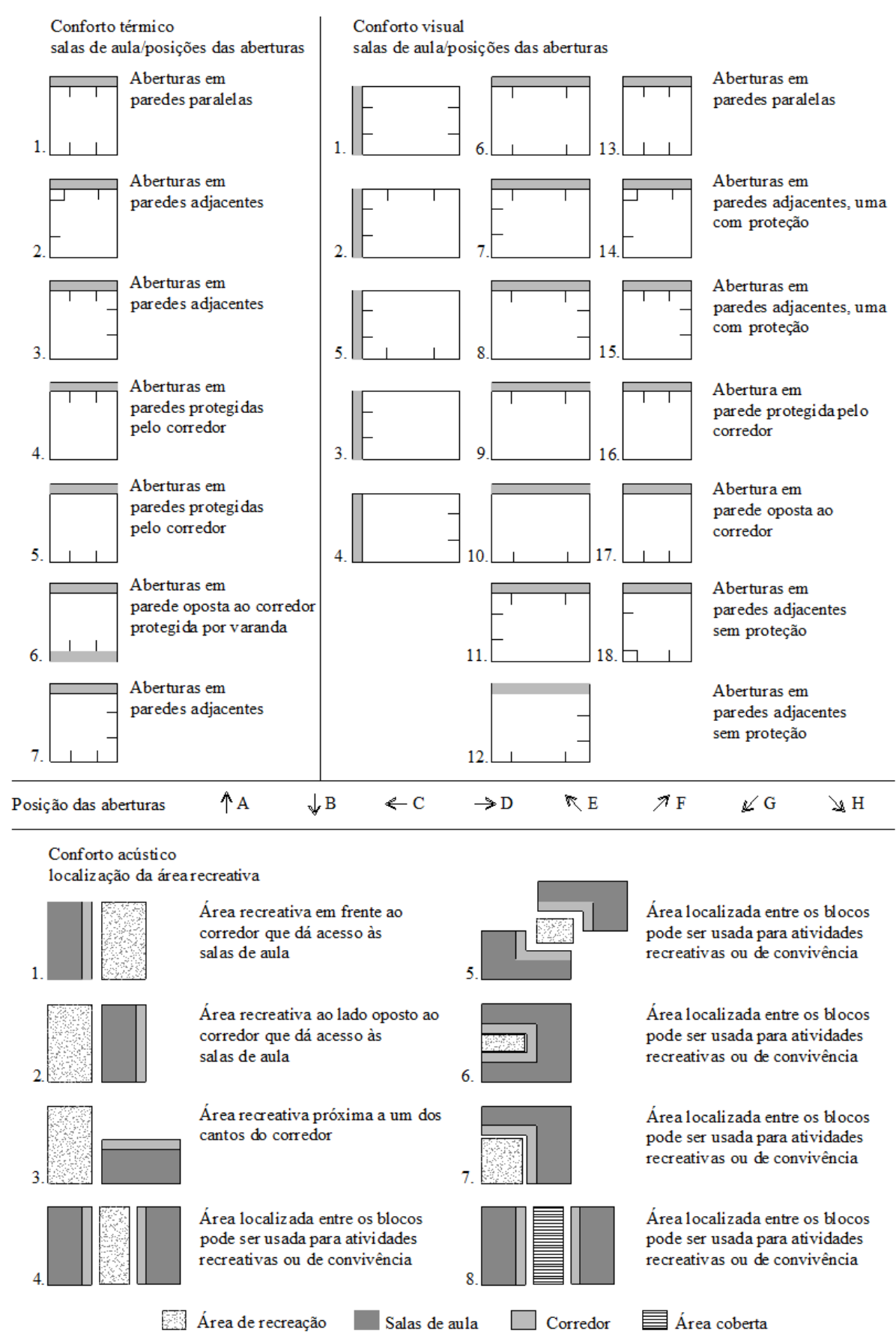

Figura 1. Tipologias, Método de avaliação de conforto ambiental. Fonte: Graça \& Kowaltowski (2004) 

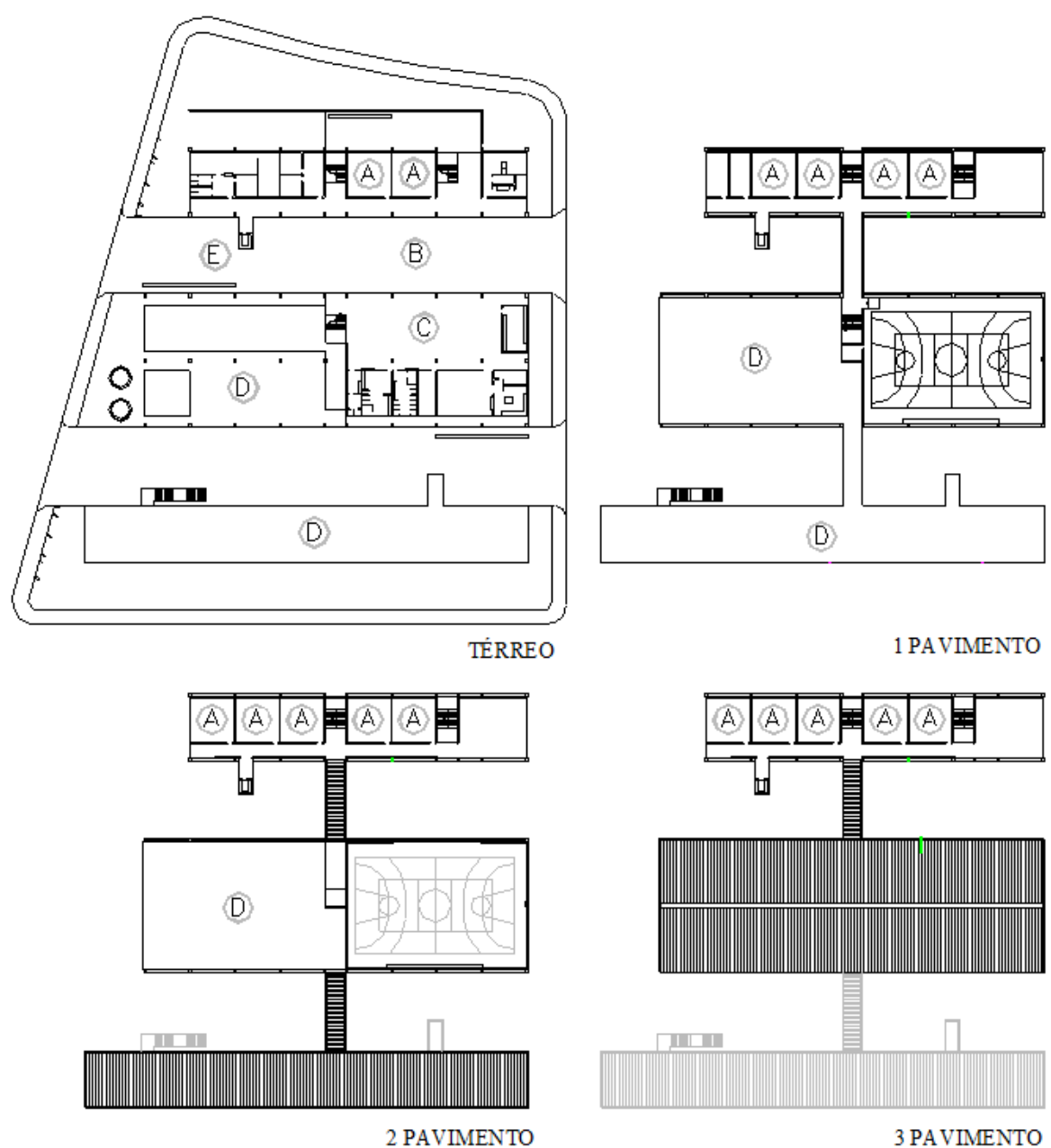

\section{LEGENDA}
A Sala de aula
(B) Pátio (C) Recreio coberto

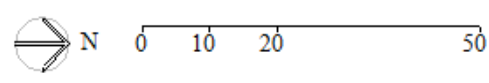

Cálculo inicial da solução inferior:

CONFORTO TÉRMICO:

Tipologia de 16 salas " 1 ", com orientação " $C$ " = grau de pertinência $0,08=>(0,08 \times 16) / 16=0,08$ CONFORTO LUMINOSO

Tipologia de 16 salas "13", com orientação "C" = grau de pertinência $0,04=>(0,04 \times 16) / 16=0,04$ CONFORTO ACÚSTICO

Tipologia de 16 salas " 4 " = grau de pertinência $0,50=>(0,50 \times 16) / 16=0,50$

Cálculo para melhoramento da solução inferior:

CONFORTO TÉRMICO:

Tipo. de 8 salas "1", com orientação "A" = 0,73/Tipo. de 8 salas "1", com orientação "B" = 0,54 $=>(0,73 \times 8)+(0,54 \times 8) / 16=0,64$

CONFORTO LUMINOSO

Tipo. de 8 salas " 1 ", com orientação " $A$ " = $0,88 /$ Tipo. de 8 salas " 1 ", com orientação "B" = 0,38 $=>(0,88 \times 8)+(0,38 \times 8) / 16=0,63$

CONFORTO ACÚSTICO

Tipo. de 8 salas " 1 " $=0,71$ /Tipo. de 8 salas " 2 " $=0,21$

$=>(0,71 \times 8)+(0,21 \times 8) / 16=0,46$

Figura 2. Solução inferior de projeto e cálculos para melhoramento dos índices. 


\subsection{Design Quality Indicator (DQI for Schools)}

O método Design Quality Indicator - DQI (DQI, 2010) é composto de quatro elementos estruturais: grade conceitual, coleta de dados, peso e "questionário" de quatro estágios. A grade conceitual é formada de três indicadores principais - funcionalidade, qualidade da construção e impacto - fundamentados nos princípios da visão tripartida firmitas, utilitas e venustas de mais de 2000 anos, proposta por Vitruvius. 0 indicador "funcionalidade" abrange o arranjo, a quantidade e a inter-relação de espaços e como o edifício é projetado para ser utilizado. 0 indicador "qualidade da construção" determina as características construtivas e de execução do edifício, ou seja, quão bem ele foi construído, sua estrutura, seus acabamentos, seus sistemas de engenharia e a coordenação de todos. Já o indicador "impactos do edifício" determina a possibilidade do edifício de agradar, intrigar e criar um senso de localização e pertencimento, inspirando a comunidade local e seu ambiente. Inclui a contribuição do projeto para a arte e para a ciência da construção e da arquitetura. A coleta de dados é constituída por dez atributos, incluídos nos indicadores principais (Tabela 2). Pelo seu formato é denominado de questionário, em forma de uma lista de indicações de avaliação.

Tabela 2. Atributos do método DQI for Schools. Fonte: DQI (2010).

\begin{tabular}{ll}
\hline Indicador DQI & Atributos \\
\hline Funcionalidade & Uso; Acesso; Espaço \\
$\begin{array}{l}\text { Qualidade } \\
\text { Construção }\end{array}$ & Desempenho; Sistemas de Engenharia; Construção \\
Impacto & $\begin{array}{l}\text { Forma \& Materiais; Ambiente Interno; Integração Social e Urbana; Inovação e } \\
\text { Características }\end{array}$ \\
\hline
\end{tabular}

O mecanismo de peso apresenta-se em forma de indicações pontuadas, sendo que a impressão/entendimento do arquiteto para cada item varia de "discordo fortemente" a "concordo fortemente", tendo a opção "não aplicável" e "não sei", numa escala de sete pontos. 0 método se mostra amigável no sentido de que cada item possui uma caixa de diálogo (best pratice tip) contendo uma explicação sumarizada.

Em cada indicador principal as características deverão ser pontuadas e, por fim, cada indicador terá também sua avaliação. Por exemplo, para o indicador "funcionalidade", as características acesso, espaço e uso deverão ser pontuadas de forma que o total contabilize 15 pontos de acordo com a impressão de importância que o respondente tem em relação a elas, independente de uma característica receber " 0 " e outra "15". Assim que a avaliação das características se encerra, os indicadores devem ser avaliados da mesma forma, no total de 15 pontos, demonstrando assim qual aspecto terá maior peso na avaliação. Ainda, é sugerido que o respondente indique três aspectos que gostou mais e menos no projeto ou construção.
Segundo o conceito do método, a pontuação dos resultados pode conferir ao projeto três estágios de qualidade: Fundamental, Adição de Valor ou Excelência. 0 ponto "Fundamental" abrange os fatores básicos e essenciais que um edifício deve contemplar para existir. O ponto "Adição de Valor" trabalha com aspectos que podem ser adicionados ao que se considera básico, aumentando a qualidade do edifício. Já o ponto "Excelência" une os três princípios, fazendo o edifício funcionar como um todo e garantindo qualidades excepcionais a ele (Tabela 3).

De acordo com os desenvolvedores do método, é recomendado que haja um facilitador durante o processo de avaliação e, em uma situação ideal, o líder (que responderá o questionário) seja um membro da equipe de projetistas. As pessoas que irão responder o questionário (tipicamente entre 5 a 15 pessoas) devem estar envolvidas de alguma forma com o projeto, tais como: clientes, membros da equipe de projetistas, usuários e contratantes. Ainda, segundo o desenvolvedor da ferramenta, as diferentes respostas dos grupos de avaliadores proporcionam diferentes visões e encorajam a comunicação entre os participantes. Não há nenhuma indicação de importância pré-estabelecida, ou ponderação 
para os indicadores. Não são efetuadas análises

estatísticas destas avaliações.

Tabela 3. Relação entre estágios de qualidade, princípios e atributos do DQI. Fonte: DQI (2010).

\begin{tabular}{|c|c|c|}
\hline Fundamental & Adição de Valor & Excelência \\
\hline Impacto & Impacto + Funcionalidade & $\begin{array}{l}\text { Impacto + Funcionalidade+ Qualidade } \\
\text { da Construção }\end{array}$ \\
\hline Paisagismo & Contribuição cívica & Valor do usuário \\
\hline Qualidades espaciais & Convivência & Inovação \\
\hline Forma externa & Eloqüência & Visão \\
\hline Iluminação artificial & Claridade da expressão & \\
\hline Iluminação natural & Composição & \\
\hline \multicolumn{3}{|l|}{ Acústica } \\
\hline \multicolumn{3}{|l|}{ Acabamento } \\
\hline Funcionalidade & $\begin{array}{l}\text { Funcionalidade + Qualidade da } \\
\text { Construção }\end{array}$ & \\
\hline Aspectos da implantação & Adaptabilidade & \\
\hline Acessibilidade & Saúde e segurança & \\
\hline Comunidade/privacidade & Facilidade de manutenção & \\
\hline $\begin{array}{l}\text { Facilidade no } \\
\text { gerenciamento }\end{array}$ & Integração & \\
\hline \multicolumn{3}{|l|}{ Conectividades } \\
\hline \multicolumn{3}{|l|}{ Possibilidades do espaço } \\
\hline \multicolumn{3}{|l|}{ Eficiência da circulação } \\
\hline \multicolumn{3}{|l|}{ Pedagogia } \\
\hline Qualidade da construção & $\begin{array}{l}\text { Qualidade da construção + } \\
\text { Impacto }\end{array}$ & \\
\hline Eficiência estrutural & Qualidade dos materiais & \\
\hline Durabilidade & Elegância estrutural & \\
\hline Acabamentos & Controle do usuário & \\
\hline Acústica & Identidade & \\
\hline \multicolumn{3}{|l|}{ Iluminação natural } \\
\hline \multicolumn{3}{|l|}{ Iluminação artificial } \\
\hline Conforto térmico & & \\
\hline
\end{tabular}

0 "questionário" de quatro estágios diz respeito aos quatro momentos diferentes do processo de construção: Programa, Projeto, Ocupação e Avaliação Pós-Ocupação. No primeiro ele é utilizado para estabelecer as necessidades dos usuários em geral, de alunos, pais, professores, funcionários e membros da comunidade e chegar a um consenso sobre as prioridades que deverão integrar o programa de necessidades.
O segundo tipo é elaborado para o uso durante o estágio de projeto, quando há desenhos, modelos, visualizações em $3 \mathrm{D}$ do edifício disponíveis. Esta versão do DQI ajuda a checar se o projeto está progredindo e se está respondendo às necessidades originais levantadas no programa de necessidades. Esta versão pode ser utilizada mais de uma vez para 
permitir diversas modificações de informações no projeto.

0 método é conhecido pelo formato que estimula o diálogo e a troca de idéias entre os participantes da avaliação. Alguns autores já apresentaram críticas em relação a este tipo de mecanismo (MARKUS, 2003; THOMSON et al., 2003; DEWULF \& VAN MEEL, 2004), embora nenhuma das críticas feitas até então se dirigem ao método específico para projetos de edificações escolares. Portanto, neste trabalho procurou-se aplicá-lo em um projeto da amostra e reunir as observações já realizadas anteriormente com os resultados da análise.

Para esta análise foi utilizado o método DQI para o Projeto de Edificações Escolares (DQI for Schools) aplicada ao projeto da EE Conjunto habitacional Campinas E1-B. Em relação à estrutura do método, notou-se que aspectos do ambiente a serem avaliados surgiram nos três indicadores de qualidade (Funcionalidade, Qualidade da Construção e Impacto) com enfoques diferenciados para cada indicador. As indicações descritas de maneira subjetiva são complementadas com indicações de verificação de normas e regulamentações. Durante a avaliação notou-se que a coleta de dados para as indicações subjetivas abrange atividades diversas como a necessidade de dialogar com outros participantes do processo, observações da planta do projeto (ou até mesmo outras representações do projeto) e suposições sobre como o ambiente irá funcionar.

A análise sobre a quantidade e qualidade de respostas para cada atributo revelou que em nenhuma questão houve uma resposta "concordo fortemente" e a quantidade de respostas "discordo fortemente" e "não sei" foram grandes em comparação aos outros níveis de qualificação (Tabela 4). Sobre os altos índices de discordância, confirma-se a impossibilidade de aplicar um método estrangeiro em projetos de escolas brasileiras sem que haja uma adaptação em todos os índices e atributos da grade conceitual. Esta adaptação refere-se tanto à regionalização dos valores de referência quanto aos valores conceituais de cada contexto analisado. As indicações de normas das ferramentas a serem utilizadas dentro de casa atributo do método também variam dependendo da região. Algumas adaptações são mais óbvias, como a utilização de normas de acessibilidade e de áreas mínimas de ambientes referentes à região de estudo. Outras adaptações referem-se às ferramentas e técnicas habituais dos profissionais.

Tabela 4. Quantidade e qualidade das respostas para cada atributo, DQI.

\begin{tabular}{lccccccccc}
\hline Atributos & DF & D & TD & TC & C & CF & N/A & N/S & Total \\
\hline Acesso & 6 & 1 & 1 & 1 & 2 & 0 & 0 & 1 & 12 \\
Espaço & 5 & 3 & 2 & 3 & 0 & 0 & 0 & 1 & 14 \\
Uso & 5 & 2 & 1 & 0 & 0 & 0 & 2 & 1 & 11 \\
Desempenho & 2 & 0 & 2 & 2 & 2 & 0 & 1 & 6 & 15 \\
Serviços de engenharia & 5 & 0 & 1 & 1 & 0 & 0 & 1 & 5 & 13 \\
Construção & 6 & 0 & 2 & 2 & 1 & 0 & 1 & 2 & 14 \\
A escola na comunidade & 0 & 2 & 0 & 2 & 1 & 0 & 0 & 2 & 7 \\
Dentro da escola & 8 & 1 & 2 & 0 & 0 & 0 & 0 & 1 & 12 \\
Formas e materiais & 6 & 1 & 0 & 0 & 0 & 0 & 0 & 0 & 7 \\
Características e inovação & 6 & 0 & 1 & 1 & 0 & 0 & 0 & 0 & 8 \\
\hline Total & 49 & 10 & 12 & 12 & 6 & 0 & 5 & 19 & 113 \\
\hline
\end{tabular}

Legenda: (DF) Discordo fortemente, (D) Discordo, (TD) Tendo a concordar, (C) Concordo, (CF)

Concordo fortemente, (N/A) Não aplicável, (N/S) Não sei. 
Para a aplicação do método foram utilizadas como documentos duas perspectivas e a planta do projeto da escola. Os resultados finais são representados em formato de gráfico de barras que mostram as diferenças entre as pontuações alcançadas e o máximo possível a ser atingido para cada atributo e indicador avaliado. Os resultados mostram um grande distanciamento entre a pontuação alcançada e a importância do atributo "Usos". Isso mostra a necessidade de avaliar todos os aspectos pertencentes ao atributo (como a relação entre as necessidades dos usuários e as qualificações espaciais, incluindo estudos sobre o método pedagógico utilizado na escola), já que, em uma possível adaptação do método para outro contexto, ele continuará tendo uma importância de destaque (Figura 3).

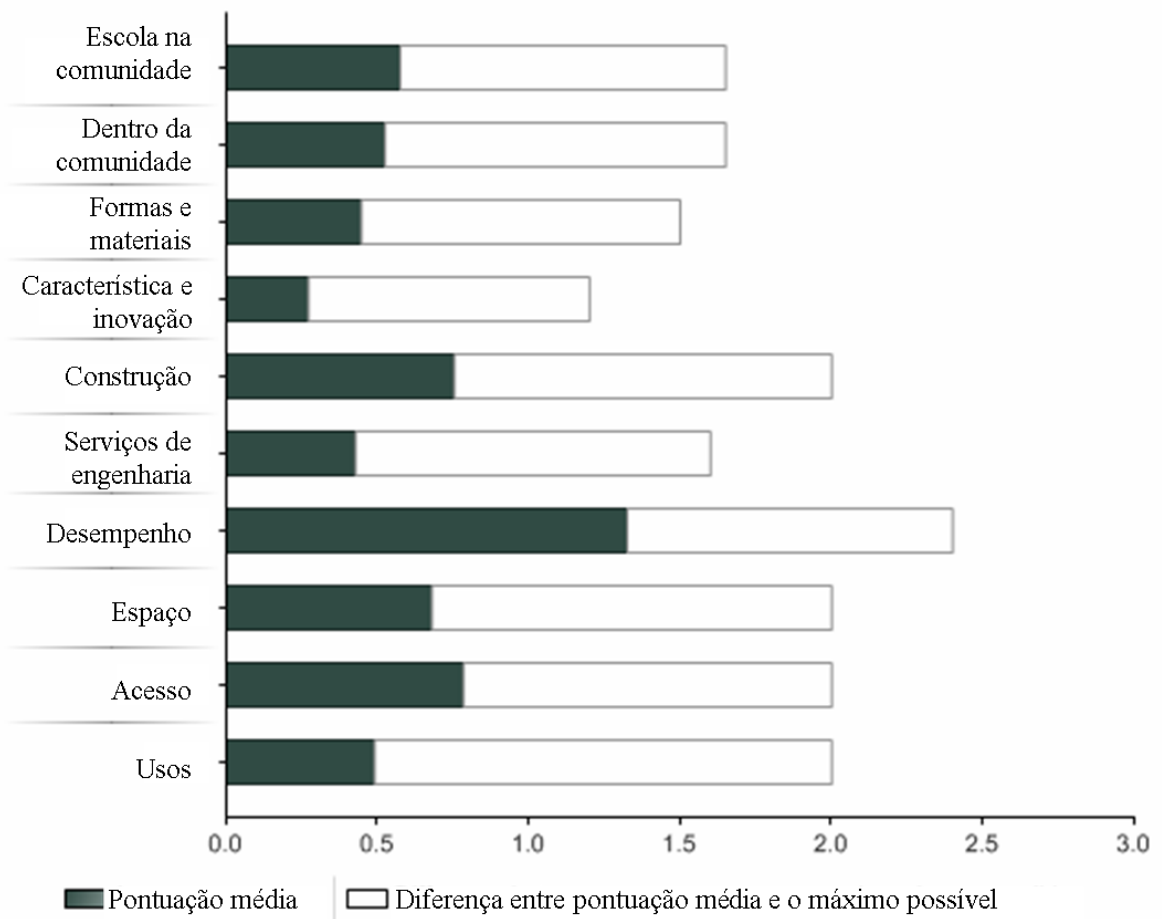

Figura 3. Resultados da aplicação do método DQI. Diferença entre a pontuação média e o máximo possível a ser alcançado.

\subsection{Comparative Floorplan Analysis}

0 método Comparative floorplan-analysis (VOORDT, VRIELINK, WEGEN, 1997) funciona como comparação de um conjunto de plantas de edifícios de mais ou menos mesma função, sempre em escala decrescente - implantação, edifício, ambientes - procurando similaridades e diferenças entre modelos de relações espaciais. Então, deve-se entender porque estas diferenças entre soluções de projeto ocorrem, relacionando as alternativas de projeto aos dados coletados de avaliações pós-ocupações (APOs). Esta relação oferece esclarecimentos sobre os pontos relevantes de decisão e (des)vantagens das variações de projeto para uso e percepção. 0 processo é de natureza interativa. Por um lado a pesquisa é guiada por hipóteses, questões e noções dos projetistas e seus clientes, dados das APOs e revisão da literatura. Por outro lado, as avaliações das plantas por si próprias geram idéias e hipóteses as quais podem ser checadas nas outras fontes.

A relação matricial entre comparação de variantes tipológicas e sistemas sociais pode fazer surgir critérios de valores expressos em diferentes soluções espaciais. A utilização do método CFA pode ser vista como uma importante adição aos métodos tradicionais de avaliação de projetos reconhecidos cientificamente, já que combina a classificação de parâmetros com a análise formal do projeto. A importância da integração com a avaliação pós-ocupação surge da impossibilidade de mensurar e coletar certos aspectos, como cores, utilização de materiais, mobiliários, apenas analisando as plantas. Pela sua estrutura, 
portanto, pode ser parte constituinte de avaliações mais completas do edifício, ou mesmo, incorporar em seu mecanismo ferramentas de análise morfológica e de estrutura social como Space Syntax de Hillier e Hanson (1984).

Na seleção da amostra de edifícios escolares para a aplicação deste método utilizou-se o critério de trabalhar somente com projetos considerados de difícil desenvolvimento causados por terrenos exíguos ou com formatos irregulares, conforme a publicação da FDE (FDE, 2006). As escolas a serem comparadas também deveriam possuir dimensões semelhantes e, para isso, utilizou-se como critério o mesmo número de salas. As escolas selecionadas foram: EE Jardim Ataliba Leonel/Pedro de Moraes Victor e EE Jardim Umuarama (Figura 4).

A matriz de valores foi elaborada a partir de resultados de entrevistas realizadas com os profissionais que desenvolveram os projetos destas escolas e durante a aplicação da ferramenta foi ajustada (Tabela 5). Foram inseridos os aspectos: topografia, localização do terreno, identidade, conforto acústico e flexibilidade. A avaliação das duas plantas gerou, além da matriz de valores, uma tabela de comparação entre os aspectos espaciais e funcionais das duas escolas (Tabela 6).

Tabela 5. Matriz de relacionamento entre aspectos espaciais e funcionais e valores e metas do projeto avaliação de projetos de edifícios escolares da FDE.

\begin{tabular}{|c|c|c|c|c|c|c|c|c|c|c|}
\hline Valores & & 1 & 2 & 3 & 4 & 5 & 6 & 7 & 8 & 9 \\
\hline $\begin{array}{l}\text { Níveis } \quad \text { de } \\
\text { avaliação }\end{array}$ & Aspectos funcionais & & & & & & & & & \\
\hline \multirow[t]{3}{*}{ Implantação } & Paisagismo & & + & & ++ & + & & ++ & ++ & \\
\hline & Localização terreno & + & ++ & ++ & & & & ++ & & + \\
\hline & Topografia & & & + & & & + & & + & \\
\hline \multirow[t]{6}{*}{ Edifício } & Acessibilidade & & & ++ & & & & ++ & & \\
\hline & Acesso & & & ++ & & & & ++ & + & \\
\hline & Circulação & & + & ++ & & & + & & & \\
\hline & Orientação & & & ++ & ++ & & ++ & & ++ & \\
\hline & Proporção & + & & & & & & & ++ & \\
\hline & Setorização & & ++ & ++ & & + & & ++ & & \\
\hline \multirow[t]{6}{*}{ Ambientes } & Aberturas & & & ++ & ++ & + & ++ & + & ++ & \\
\hline & Dimensões & & + & ++ & + & + & + & & ++ & ++ \\
\hline & $\begin{array}{l}\text { Dispositivos de proteção } \\
\text { solar }\end{array}$ & & & & ++ & & + & & ++ & \\
\hline & Hierarquia & & ++ & ++ & & & & & & \\
\hline & Padronização & & + & & & & ++ & & ++ & \\
\hline & Flexibilidade & & + & + & & & & & + & + \\
\hline Legenda & \multicolumn{10}{|c|}{$\begin{array}{l}\text { ++ Relações baseadas nas opiniões de arquitetos projetistas das escolas da } \\
\text { FDE } \\
\text { + Relações originadas a durante a aplicação da ferramenta }\end{array}$} \\
\hline $\begin{array}{l}\text { Legenda dos } \\
\text { valores }\end{array}$ & $\begin{array}{l}\text { 1. Identidade } \\
\text { 2. Arq. e pedagogia } \\
\text { 3. Funcionalidade } \\
\text { 4. Conf. térmico e visual } \\
\text { 5. Conf. acústico } \\
\text { 6. Economia } \\
\text { 7.Segurança } \\
\text { 8. Estética } \\
\text { 9. Temporalidade }\end{array}$ & & & & & & & & & \\
\hline
\end{tabular}



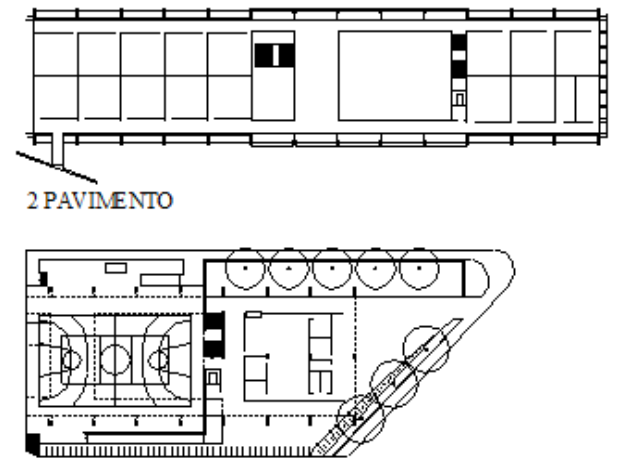

TÉRREO

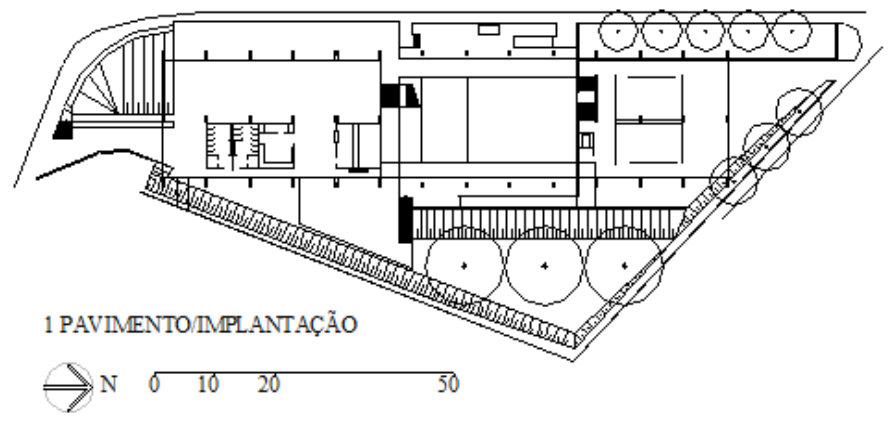

(a)

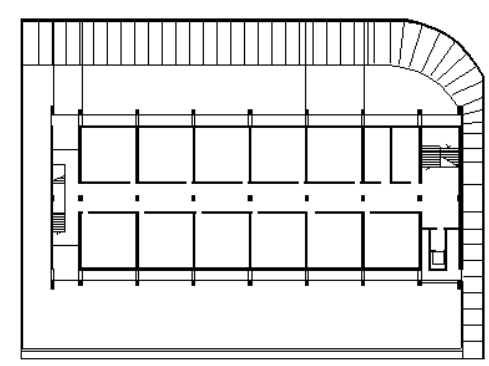

TÉRREO
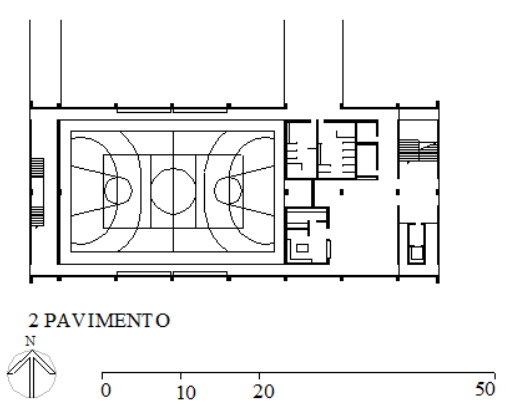

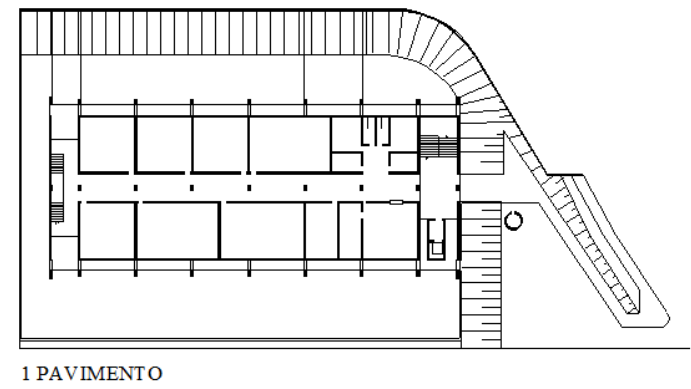

1 PAVIMENTO

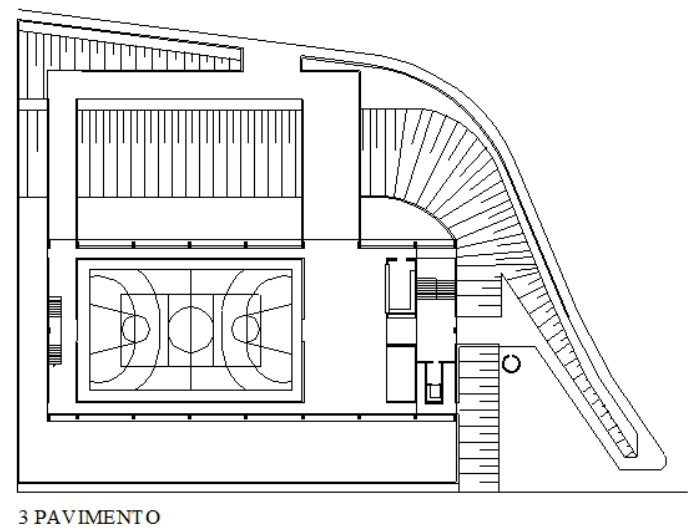

(b)

Figura 4. (a)Planta da escola EE Jardim Ataliba Leonel; (b) Jardim Umuarama. Fonte adaptada: FDE (2006). 
Tabela 6. Comparação de aspectos espaciais e funcionais entre a EE Jardim Ataliba Leonel/Pedro Moraes Victor e EE Jardim Umuarama.

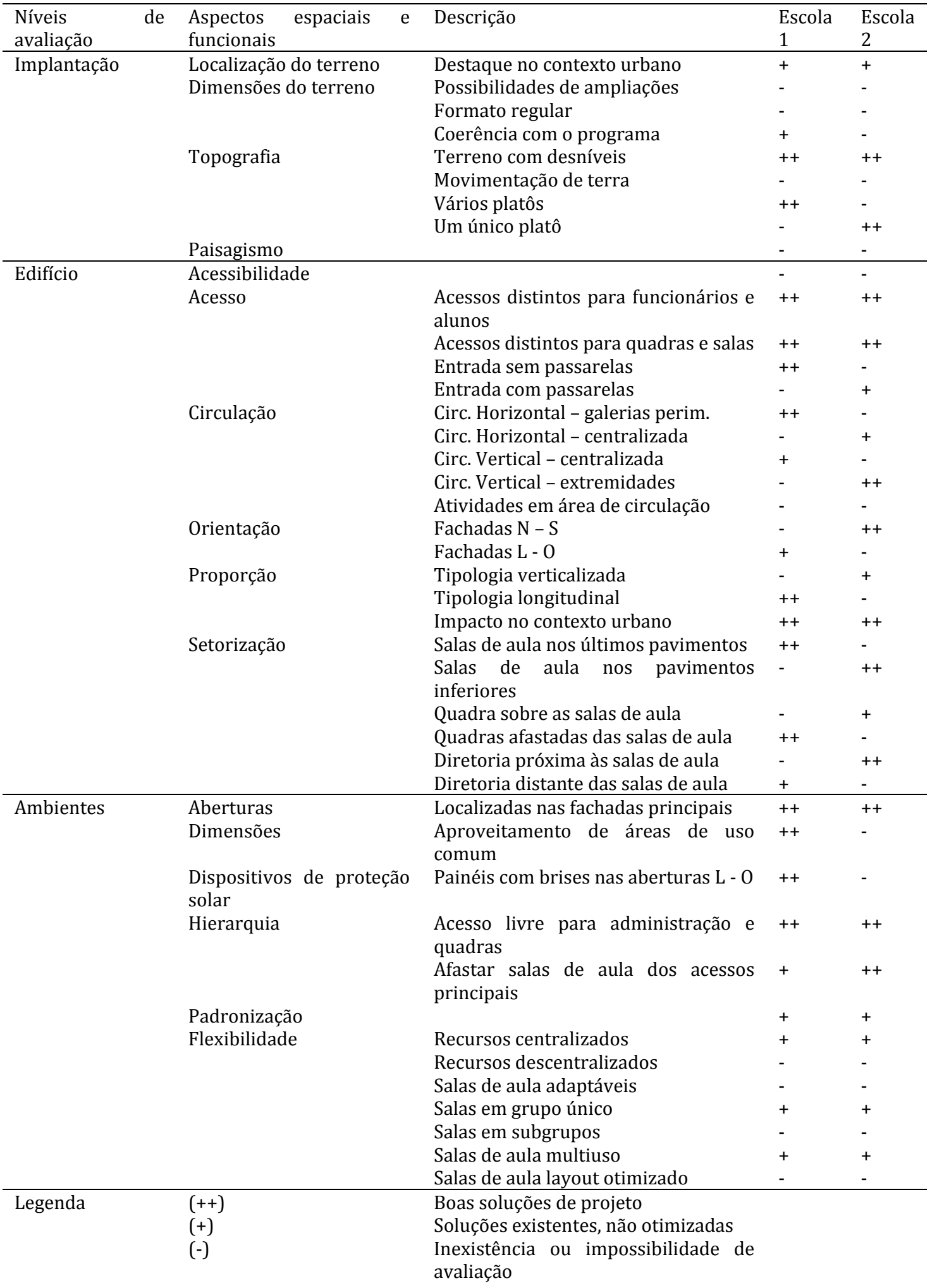




\section{DISCUSSÃO DOS RESULTADOS}

A análise do método DQI for Schools sugere modificações principalmente no que se trata de seu sistema de pontuação. A natureza das atividades que envolvem consenso sobre a qualidade de aspectos subjetivos diverge da coleta de dados de indicações objetivas, já que a verificação à norma indica se o projeto está dentro dos padrões ou não. Os indicadores do método unem os aspectos subjetivos e objetivos para um mesmo atributo e eles podem ser capturados separadamente. Desta maneira, a pontuação dos dados em escala de sete pontos pode se tornar imprecisa para respostas "sim" ou "não" e restritas em avaliações que englobam vários critérios subjetivos e contam com o acordo entre os avaliadores. Também, opções do tipo "não saber" em questionários de pontuação podem se tornar alternativas que não incentivam a busca de informações para a avaliação do projeto.

Outro aspecto refere-se às informações contidas no objeto de análise. Para responder questões referentes aos atributos técnicos inseridos no indicador Qualidade da Construção sentiu-se necessidade de informações mais detalhadas sobre o projeto. Seria interessante que o método definisse as representações gráficas de projetos a serem utilizados durante a aplicação (planta, corte, elevação, perspectivas 3D, 4D, maquetes, etc.). Comparações entre bons projetos que agrupem um conjunto de características ideais e o projeto avaliado podem ser realizadas. A experiência profissional e os resultados de avaliações pós-ocupação em ambientes similares ao projeto avaliado podem ser incluídos neste tipo de aplicação.

O DQI for Schools pode ser caracterizado, principalmente, como um método valioso para o desenvolvimento do programa arquitetônico, devido ao seu caráter generalista e enriquecedor do diálogo. Também pode formar conjunto de princípios de análise de projetos, já que a grade conceitual é completa e fundamentada. A apresentação dos resultados caracteriza muito mais um resumo de todo o trabalho de avaliação do que um instrumento de realimentação de projeto.
A análise do "Método de avaliação de conforto ambiental de projetos escolares - otimização multi-critérios" também revelou a necessidade de revisões. A seleção de terrenos poderia contar com análise da topografia, além da razão das dimensões. Foi necessário digitalizar todas as plantas da amostra e criar blocos no programa CAD referentes aos graus de pertinência e às orientações para facilitar a utilização da ferramenta. Tendo em vista que atualmente os escritórios de arquitetura contam com todos os projetos digitalizados, esta adaptação é viável. Para que o arquiteto desenvolva um projeto de edifício escolar de qualidade são necessários mais requisitos para análise, além dos aspectos de conforto ambiente. 0 método pode ser incluído em algum outro método com grade conceitual generalizada.

O método Comparative floorplan analysis se mostrou interessante por permitir que o processo de análise seja feito de forma iterativa e utilizando elementos gráficos. A estrutura do método abre amplas possibilidades para que a comparação inclua resultados de pesquisas e experiência dos avaliadores (arquitetos). Um ponto desfavorável, para o objetivo desta pesquisa, é que a análise das plantas estrutura os resultados em forma de tipologias de projeto. 0 processo de qualificação destas tipologias também poderia ser mais definido se houvesse um plano-zero de referência. Outro ponto desfavorável é a limitação da legibilidade de alguns aspectos em planta, como os materiais, volume e equipamentos.

Concluindo, os métodos apresentaram vantagens e desvantagens sob cada aspecto analisado. O DQI for Schools apresenta uma grade conceitual completa, e estimula a busca de referências e o diálogo sobre as condições do projeto. 0 Comparative floorplan analysis mostra que o uso de elementos gráficos estimula a geração de novas idéias, além de utilizar a linguagem que o profissional está habituado. 0 método "otimização multi-critérios" mostra que a precisão na quantificação dos resultados agiliza a avaliação e não abre lacunas para possíveis dúvidas sobre a qualidade do julgamento. 
Tabela 7. Resultados das aplicações dos métodos e da ferramenta.

\begin{tabular}{|c|c|}
\hline Fatores & DQI \\
\hline $\begin{array}{l}\text { Fase ideal de } \\
\text { aplicação }\end{array}$ & Programa e projeto. \\
\hline $\begin{array}{l}\text { Grade } \\
\text { conceitual }\end{array}$ & $\begin{array}{l}\text { Permite inserção de novos } \\
\text { conceitos. É abrangente. }\end{array}$ \\
\hline $\begin{array}{l}\text { Sistema de } \\
\text { pontuação }\end{array}$ & $\begin{array}{l}\text { Quantitativo. Escala de } \\
\text { preferências/pesos. }\end{array}$ \\
\hline Estrutura & Longa \\
\hline $\begin{array}{l}\text { Apresentação } \\
\text { dos resultados }\end{array}$ & Pouco eficiente. Gráficos. \\
\hline Revisões & Sistema de pontuação. \\
\hline $\begin{array}{l}\text { Os resultados } \\
\text { confirmam a nece } \\
\text { de um método de a } \\
\text { que inclua em } \\
\text { específicos para a } \\
\text { objetivos e subjeti } \\
\text { arquitetos exige } \\
\text { informações dura } \\
\text { projeto, sendo inte } \\
\text { elementos gráficos } \\
\text { Um modelo que in }\end{array}$ & $\begin{array}{l}\text { finais das três aplicações } \\
\text { essidade do desenvolvimento } \\
\text { avaliação de projetos escolares } \\
\text { sua estrutura elementos } \\
\text { a coleta e análise de dados } \\
\text { vos. A prática profissional dos } \\
\text { um retorno rápido de } \\
\text { ante o desenvolvimento do } \\
\text { eressante que sejam incluídos } \\
\text { s e dimensionais no sistema. } \\
\text { aclua a visualização do plano- }\end{array}$ \\
\hline
\end{tabular}

Otimização multi-critérios

Projeto finalizado.

Não permite inserção de novos conceitos. Focada nos aspectos de conforto.

Quantitativo. Índices.

Concisa

Flexível

Eficiente, tendo em vista a proposta. Índices.

Relaciona resultados à análise gráfica.

Método de exclusão de Mais testes. terrenos.

zero (o modelo de referência) também é válido. A análise da grade conceitual mostrou que não é possível aplicar, sem adaptações, métodos que estejam levando em consideração valores e princípios de ambientes escolares estrangeiros. 0 método para projetos de edificações escolares do Estado de São Paulo requer um conjunto de requisitos específicos de projeto para tal realidade, além de um sistema inserido nos padrões de custo-benefício das políticas do Estado e das práticas da FDE.

\section{REFERÊNCIAS}

BLOWER, H.C.S.; AZEVEDO, G.A.N. Avaliação pós-ocupação em creche institucional do município do Rio de Janeiro: uma experiência no lugar da educação infantil. Revista Gestão e Tecnologia de Projetos, v.5; 99130, EESC - USP, 2010.

CHERRY, E., Programming for design: from theory to pratice. New York: John Wiley \& Sons, Inc, 1999.

COLE, R. J. Building environmental assessment methods: redefining intentions and roles. Building Research and Information, 33:5, 455-467, 2005. doi:10.1080/09613210500219063

DELIBERADOR, M.S. O processo de projeto de arquitetura escolar no Estado de São Paulo: caracterização e possibilidades de intervenção. Dissertação (Mestrado em Engenharia Civil). UNICAMP - Universidade Estadual de Campinas. Campinas, São Paulo, 2010.

DESIGN QUALITY INDICATOR (DQI). The DQI tool. Disponível em http://www.dqi.org.uk acessado em Dezembro de 2010.

DEWULF, G.; VAN MEEL, J. Sense and nonsense of measuring design quality. Building Research and Information. 2004, v32, n3, pp 247-250.

DUDEK, M. Schools and Kindergartens - a design manual. Berlin: Birkäuser, 2007. 
FCAV - Fundação Vanzolini. Referencial técnico de certificação “Edifícios do setor de serviços Processo AQUA". Escritórios e Edifícios escolares. Outubro de 2007.

FIGUEIREDO, F.G. Processo de Projeto Integrado visando à melhoria do desempenho ambiental de edificações: levantamento, análise e comparação de dois estudos de caso. Dissertação (Mestrado em Engenharia Civil). UNICAMP - Universidade Estadual de Campinas. Campinas, São Paulo, 2009.

FORD, A.; HUTTON, P. A sense of entry. Austrália: Melina Deliyannis, 2007.

FUNDAÇÃO PARA O DESENVOLVIMENTO DA EDUCAÇÃO (FDE). Arquitetura escolar paulista estruturas pré-fabricadas, São Paulo, Diretoria de obras e serviços, 2006, p 336.

GRAÇA, V.A.Z. da; KOWALTOWSKI, D.C.C.K. Metodologia de avaliação de conforto ambiental de projetos escolares usando o conceito de otimização multicritério. Revista ambiente construído, v. 4, n. 3, 2004, pp. 19 - 35 .

HILLER, B.; HANSON, J. The social logic of space. Cambridge: Cambridge University Press, 1984. doi: $10.1017 / C B 09780511597237$

KOWALTOWSKI, D.C.C.K; CELANI, M.G.C; MOREIRA, D.C.; PINA, S.AM.; RUSCHEL, R.C.; SILVA,V.G. da; LABAKI, L.C. \& PETRECHE,J.R.D. Reflexão sobre metodologias de projeto arquitetônico. Revista Ambiente Construído. Porto Alegre, v.6, n.2, p. 07-19, abr./jun. 2006.

KOWALTOWSKI, D.C.C.K. Arquitetura Escolar: o projeto do ambiente de ensino. São Paulo: Oficina de textos, 2011.

KOWALTOWSKI, D.C.C.K.; MOREIRA, D.C.; PETRECHE, J.R.D; FABRÍCIO M. o processo de projeto em arquitetura: da teoria a tecnologia. Ed. Oficina de Textos, dez. 2011, ISBN 978-85-7975-033-5, p.504.

KOWALTOWSKI, D.C.C.K.; BORGES FILHO, F.; LABAKI, L.C.; RUSCHEL, R.C.; BERTOLI, S.R. \& PINA, S.A.M.G. Melhoria do conforto ambiental em edificações escolares estaduais de Campinas - SP. Relatório Científico/ Fapesp. Campinas, SP: UNICAMP, 2001.

KRUGER, C. \& CROSS, N., Solution driven versus problem driven design: strategies and outcomes. Design Studies, v.27, pp. 527-548, 2006. doi:10.1016/j.destud.2006.01.001

LIPPMAN, P.C. Evidence-based design of elementary and secondary schools. A responsive approach to creating learning environments. John Wiley \& Sons, Inc. New Jersey, 2010.

MARKUS, T.A. Lessons from the design quality indicator. Building Research and Information. 2003, v31, n5, pp399-405.

MOREIRA, D. De Carvalho \& KOWALTOWSKI, D.C.C.K. Discussão sobre a importância do programa de necessidades para a qualidade no processo de projeto em arquitetura. Revista ANTAC, Ambiente Construído, jun 2009, v. 9, n. 2 pp. 31-45.

MÜLLER, C.M. Espaços de ensino-aprendizagem com qualidade ambiental: o processo metodológico para elaboração de um anteprojeto. Dissertação. (Mestrado em Arquitetura e Urbanismo). Faculdade de Arquitetura e Urbanismo da Universidade de São Paulo. São Paulo, 2007.

NAIR, P. \& FIELDING, R. The language of school design. Design patterns for the 21th century school. 2ed. Nacional Clearinghouse for Educacional Facilities. Índia, 2005.

ORNSTEIN, S.W. \& MOREIRA, N.S. Evaluating School Facilities in Brazil. OECD/PEB - Program on Educational Building Department), 2008.

PIZARRO, P.R. Estudo das variáveis do conforto térmico e luminoso em ambientes escolares. Dissertação (Mestrado em Desenho Industrial). UNESP - Universidade Estadual Paulista (Faculdade de Arquitetura, Artes e Comunicação). 155p. Bauru, São Paulo, 2005.

PREISER W.F.E.; RABINOWITZ H.Z. \& WHITE E.T., Post-occupancy evaluation, New York: Van Nostrand Reinhold, 1988.

RHEINGANTZ, P. A.; AZEVEDO, G.A.N.; BRASILEIRO, A.; ALCANTRA, D.; QUEIROZ, M. Observando a qualidade do lugar: procedimentos para a avaliação pós-ocupação. Rio de Janeiro: Universidade Federal do Rio de Janeiro, Faculdade de Arquitetura e Urbanismo, 2009, 117 p. Disp. em:<http://www.fau.ufrj.br/prolugar/arq_pdf/livros/obs_a_qua_lugar.pdf>. Acesso em 01 de maio de 2011. 
SANOFF, H. School buildings assessment methods. Nacional Clearinghouse for Educacional facilities. Washington, DC., 2001.

TARALLI, C.H., Demandas Sociais e escola pública. In: NUTAU - Demandas Sociais, Inovações Tecnológicas e a Cidade. Seminário Internacional, 11-15 de Outubro, São Paulo, Brasil. 2004. Anais Digitais, 2004.

THOMSON, D.S.; AUSTIN, S.A.; DEVINE-WRIGHT, H.; MILLS, G.R. Managing value and quality in design. Building Research and Information. 2003, v31, n5, pp334-345.

VOORDT, T. J. M. van der; VRIELINK, D.; WEGEN, H. B. R. van. Comparative floorplan analysis in programming and architectural design. Design Studies, 18, 67-88, 1997. doi:10.1016/S0142694X(96)00016-6

WERTHEIN, J., É preciso mais que um ensino médio. O Estado de São Paulo. São Paulo, 20 de fevereiro, 2010.

\section{AGRADECIMENTOS}

Os autores agradecem à FAPESP pelo apoio financeiro recebido para o desenvolvimento da pesquisa.

\section{DADOS DOS AUTORES}

(i)Doris Catharine Cornelie Knatz Kowaltowski é Professora Titular (2009) pela Universidade Estadual de Campinas. Basil.

(ii) Doutoranda em arquitetura na Universidade Estadual de Campinas. 